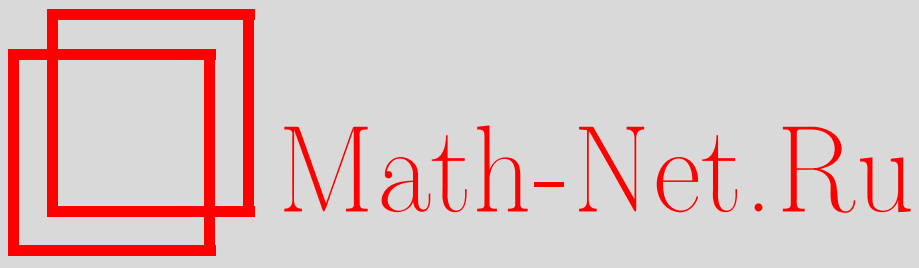

А. Н. Бахвалов, Представление непериодических функций ограниченной $\Lambda$ вариации интегралом Фурье в многомерном случае, Изв. РАН. Сер. матем., 2003, том 67, выпуск 6, 3-22

DOI: https://doi.org/10.4213/im458

Использование Общероссийского математического портала Math-Net.Ru подразумевает, что вы прочитали и согласны с пользовательским соглашением

http: //www.mathnet.ru/rus/agreement

Параметры загрузки:

IP : 52.23 .180 .231

26 апреля 2023 г., 07:36:14 
УДК 517.518

\author{
А.Н. Бахвалов
}

\title{
Представление непериодических функций ограниченной $\Lambda$-вариации интегралом Фурье в многомерном случае
}

\begin{abstract}
Установлены достаточные условия сходимости кратного интеграла Фурье интегрируемой функции по Прингсхейму (в смысле сходимости частичных интегралов по параллелепипедам) в терминах принадлежности функции классам ограниченной $\Lambda$-вариации. Эти условия состоят в следующем: функция принадлежит классу ограниченной гармонической вариации, рассматриваемая точка регулярна, гармоническая вариация в окрестности этой точки ведет себя "хорошо", функция непрерывна по гармонической вариации на специальном подмножестве в окрестности бесконечности. Показано также, что ни одно из двух последних условий, вообще говоря, нельзя исключить.

Библиография: 10 наименований.
\end{abstract}

\section{§1. Введение}

Введем необходимые обозначения. Пусть $C$ - абсолютные постоянные, а $C(\cdot)$ величины, зависящие лишь от перечисленных в скобках параметров (не обязательно одинаковые в различных случаях).

Для промежутка $I$ на прямой через $\Omega(I)$ обозначим множество всех конечных систем попарно непересекающихся интервалов $\left\{I_{n}\right\}$ таких, что $\overline{I_{n}} \subset I$.

Пусть $I^{k}=\left(a^{k}, b^{k}\right)$. Рассмотрим функцию $f(\mathbf{x})$ на $\mathbb{R}^{m}$. При $m=1$ положим $f\left(I^{1}\right)=f\left(b^{1}\right)-f\left(a^{1}\right)$; если для любой функции $m-1$ переменных уже определено выражение $f\left(I^{1} \times \cdots \times I^{m-1}\right)$, то для функции $m$ переменных положим

$$
f\left(I^{1} \times \cdots \times I^{m}\right)=f\left(I^{1} \times \cdots \times I^{m-1}, b^{m}\right)-f\left(I^{1} \times \cdots \times I^{m-1}, a^{m}\right) .
$$

Величину $f\left(I^{1} \times \cdots \times I^{m}\right)$ будем называть смешанным приращением функщии $f$ на $I$.

Пусть множество $\{1, \ldots, m\}$ разбито на два непересекающихся множества $\alpha$ и $\beta$, состояших из $p$ и $m-p$ элементов соответственно. Будем обозначать $|\alpha|=p$, $|\beta|=m-p$. Если $\mathbf{x}=\left(x^{1}, \ldots, x^{m}\right)$, то $x^{\alpha}$-элемент $\mathbb{R}^{p}, \operatorname{cocтоящий~из~компонент~} x^{j}$, $j \in \alpha$. Для параллелепипеда $I=\bigotimes_{j=1}^{m} I^{j}$ обозначим $I^{\alpha}=\bigotimes_{j \in \alpha} I^{j}$. Через $x^{\alpha}+I^{\alpha}$ (в частности, $\mathbf{x}+I$ ) обозначим сдвиг параллелепипеда на вектор, а через $f\left(I^{\alpha}, x^{\beta}\right)$ - смешанное приращение $f$ как функции аргументов $x^{j}, j \in \alpha$, на $I^{\alpha}$ при фиксированных значениях $x^{k}, k \in \beta$.

Работа выполнена при финансовой поддержке Российского фонда фундаментальных исследований (грант № 03-01-00080), государственных программ "Ведущие научные школы" (грант № НШ-1657.2003.01) и “Молодые кандидаты” (грант № MK-1643.2003.01).

(C) А.Н. БАХвАлов, 2003 
ОПРЕДЕЛЕНИЕ 1. Неубывающая последовательность положительных чисел $\Lambda=\left\{\lambda_{n}\right\}$ задает класс функиий ограниченной $\Lambda$-вариации (класс Ватермана), если $\lambda_{n} \rightarrow \infty$ при $n \rightarrow \infty$ и $\sum_{n=1}^{\infty} \frac{1}{\lambda_{n}}=\infty$. Далее будем рассматривать только такие $\Lambda$. Множество последовательностей $\Lambda$, удовлетворяюших перечисленным условиям, будем обозначать через $\mathbb{L}$. Частичные суммы $\sum_{k=1}^{N} \frac{1}{\lambda_{k}}$ обозначим через $\Lambda(N)$, последовательность $\left\{\lambda_{k}\right\}_{k=n+1}^{\infty}-$ через $\Lambda_{n}$. Положим также $H=\{n\}$.

ОПРЕДЕЛЕНИЕ 2 . Пусть $\Lambda^{1}, \ldots, \Lambda^{m} \in \mathbb{L}$. Тогда $\left(\Lambda^{1}, \ldots, \Lambda^{m}\right)$-вариацией функции $f\left(x^{1}, \ldots, x^{m}\right)$ относительно переменных $x^{1}, \ldots, x^{m}$ по параллелепипеду $\Delta=\Delta^{1} \times \cdots \times \Delta^{m}$ называется величина

$$
V_{\Lambda^{1}, \ldots, \Lambda^{m}}^{x^{1}, \ldots, x^{m}}(f ; \Delta)=V_{\Lambda^{1}, \ldots, \Lambda^{m}}^{\mathbf{x}}(f ; \Delta)=\sup _{\left\{I_{k^{j}}^{j}\right\} \in \Omega\left(\Delta^{j}\right)} \sum_{k^{1}, \ldots, k^{m}} \frac{\left|f\left(I_{k^{1}}^{1} \times \cdots \times I_{k^{m}}^{m}\right)\right|}{\lambda_{k^{1}}^{1} \cdots \lambda_{k^{m}}^{m}} .
$$

Пусть непустое множество $\alpha \subset\{1, \ldots, m\}$ состоит из элементов $j_{1}<\cdots<j_{p}$ и $\beta=\{1, \ldots, m\} \backslash \alpha$. Через

$$
V_{\Lambda^{\alpha}}^{x^{\alpha}}\left(f ;\left(\Delta^{\alpha}, x^{\beta}\right)\right)=V_{\Lambda^{j_{1}}, \ldots, \Lambda^{j p}}^{x^{\alpha}}\left(f ;\left(\Delta^{\alpha}, x^{\beta}\right)\right)
$$

обозначим $\left(\Lambda^{j_{1}}, \ldots, \Lambda^{j_{p}}\right)$-вариацию $f$ как функции переменных $x^{j_{1}}, \ldots, x^{j_{p}}$ по $p$-мерному параллелепипеду $\Delta^{\alpha}=\Delta^{j_{1}} \times \cdots \times \Delta^{j_{p}}$ при фиксированных значениях $x^{\beta}$ остальных переменных (если $\beta$ непусто). Возникающие при этом параллелепипеды $\bigotimes_{l=1}^{p} I_{k^{j_{l}}}^{j_{l}}$ для краткости запишем как $I_{k^{\alpha}}^{\alpha}$, а произведения $\lambda_{k^{j_{1}}}^{j_{1}} \cdots \lambda_{k^{j_{p}}}^{j_{j_{p}}}-$ как $\lambda_{k^{\alpha}}^{\alpha}$.

Далее, $\left(\Lambda^{j_{1}}, \ldots, \Lambda^{j_{p}}\right)$-вариацией функиии $f\left(x^{1}, \ldots, x^{m}\right)$ относительно переменных $x^{\alpha}$ по параллелепипеду $\Delta=\Delta^{1} \times \cdots \times \Delta^{m}$ называется величина

$$
V_{\Lambda^{\alpha}}^{x^{\alpha}}(f ; \Delta)=V_{\Lambda^{j_{1}}, \ldots, \Lambda^{j_{p}}}^{x^{\alpha}}(f ; \Delta)=\sup _{x^{\beta} \in \Delta^{\beta}} V_{\Lambda^{j_{1}}, \ldots, \Lambda^{j_{p}}}^{x^{\alpha}}\left(f ;\left(\Delta^{\alpha}, x^{\beta}\right)\right) .
$$

ОПРЕДЕЛЕнИЕ 3. Величина

$$
V_{\Lambda^{1}, \ldots, \Lambda^{m}}(f ; \Delta)=\sum_{\substack{\alpha \subset\{1, \ldots, m\} \\ \alpha \neq \varnothing}} V_{\Lambda^{\alpha}}^{x^{\alpha}(f ; \Delta)}
$$

называется (полной) $\left(\Lambda^{1}, \ldots, \Lambda^{m}\right)$-вариацией функции $f\left(x^{1}, \ldots, x^{m}\right)$ по параллелепипеду $\Delta=\Delta^{1} \times \cdots \times \Delta^{m}$. Множество функций, для которых она конечна, называется классом $\left(\Lambda^{1}, \ldots, \Lambda^{m}\right)$-ограниченной вариачии и обозначается $\left(\Lambda^{1}, \ldots, \Lambda^{m}\right) \mathrm{BV}(\Delta)$. Если все последовательности $\Lambda^{j}$ совпадают и равны $\Lambda$, то для краткости будем записывать $V_{\Lambda}^{x^{\alpha}}, V_{\Lambda}, \Lambda \mathrm{BV}(\Delta)$ и $\lambda_{k^{\alpha}}$. Величину $V_{H}$ называют гармонической вариачией.

Далее предположим, что все рассматриваемые функции измеримы.

ОПРЕДЕЛЕНИЕ 4. Функция $f \in\left(\Lambda^{1}, \ldots, \Lambda^{m}\right) \mathrm{BV}(\Delta)$ называется непрерывной по $\left(\Lambda^{1}, \ldots, \Lambda^{m}\right)$-вариации, если для любого непустого множества $\alpha=$ $\left\{j_{1}, \ldots, j_{p}\right\} \subset\{1, \ldots, m\}$ и любого $j_{k} \in \alpha$ вьполнено

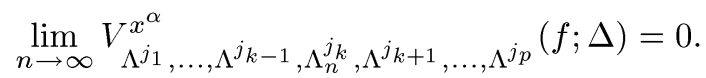

Множество таких функций обозначим через $\mathrm{C}\left(\Lambda^{1}, \ldots, \Lambda^{m}\right) \mathrm{V}(\Delta)$. 
ОПРЕДЕЛЕНИЕ 5. Точка $\mathbf{x}_{0}$ является регулярной точкой функции $f(\mathbf{x})$, если существуют и конечны $2^{m}$ пределов

$$
f\left(x_{0}^{1} \pm 0, \ldots, x_{0}^{m} \pm 0\right)=\lim _{t^{1}, \ldots, t^{m} \rightarrow+0} f\left(x_{0}^{1} \pm t^{1}, \ldots, x_{0}^{m} \pm t^{m}\right)
$$

для всевозможных комбинаций знаков. Для регулярной точки $\mathbf{x}_{0}$ обозначим

$$
f^{*}\left(\mathbf{x}_{0}\right)=\frac{1}{2^{m}} \sum f\left(x_{0}^{1} \pm 0, \ldots, x_{0}^{m} \pm 0\right) .
$$

Пусть $\boldsymbol{\delta} \in(0, \infty)^{m}$, а $\boldsymbol{\sigma} \in\{-1,1\}^{m}$. Обозначим через $(\mathbf{x}, \mathbf{x}+\boldsymbol{\sigma} \boldsymbol{\delta})$ параллелепипед $\bigotimes_{j=1}^{m} I^{j}$, где $I^{j}=\left(x^{j}, x^{j}+\delta^{j}\right)$ при $\sigma^{j}=1$ и $I^{j}=\left(x^{j}-\delta^{j}, x^{j}\right)$ при $\sigma^{j}=-1$. В качестве нормы в $\mathbb{R}^{m}$ возьмем $\|\boldsymbol{\delta}\|=\max \left|\delta^{j}\right|$.

Классы $\Lambda$ BV в одномерном случае были определены Д. Ватерманом [1], в двумерном случае - А.А. Саакяном [2], а для случая более высоких размерностей А.И. Саблиным [3], [4]. Ими были установлены следующие результаты о сходимости рядов Фурье (здесь и далее рассматриваются ряды Фурье по (кратной) тригонометрической системе).

Tеорема А (Д. Ватерман). Пусть $f \in \operatorname{HBV}([-\pi, \pi])$. Тогда в кажсдой точке ряд Фурье функиии $f$ сходится к величине $\frac{1}{2}(f(x+0)+f(x-0))$ и сходимость равномерна на любом отрезке, лежсащем внутри интервала непрерывности функиии.

Теорема В (А.А. Саакян). Пусть $f-2 \pi$-периодическая по каждому аргументу функиия на плоскости и $f \in \operatorname{HBV}\left([-\pi, \pi]^{2}\right)$. Тогда в каждой регулярной точке $\mathbf{x}$ ряд Фурье функиии $f(\mathbf{x})$ сходится по Прингсхейму к величине $f^{*}(\mathbf{x})$. Если функиия непрерывна на открытом множестве E, то сходимость равномерна на любом компакте, лежащем в Е.

Теорема С (А.И. Саблин). Пусть $f(x) \in \operatorname{HBV}\left([-\pi, \pi]^{m}\right)-$ непрерывная $2 \pi$-периодическая по каждому аргументу функиия и

$$
\lim _{\operatorname{diam} I \rightarrow 0} V_{H}(f ; I)=0
$$

равномерно по всем параллелепипедам I. Тогда ее ряд фурье равномерно сходится к ней по Прингсхейму.

Если $f(x) \in\left(\Lambda^{1}, \ldots, \Lambda^{m}\right) \mathrm{BV}\left([-\pi, \pi]^{m}\right)$ - непрерывная $2 \pi$-периодическая по каждому аргументу функиия, причем $\lambda_{k}^{q} \leqslant k$ идя любого $p \in\{1, \ldots, m\}$ справедливо условие

$$
\sum_{k=1}^{\infty} \prod_{\substack{q=1 \\ q \neq p}}^{m} \frac{1}{\lambda_{k}^{q}}=\infty
$$

то ее ряд Фурье равномерно сходится к ней по Прингсхейму.

Результат Саблина был усилен автором в работе [5], где доказана 
TEорема D. Для любой непрерывной $2 \pi$-периодической по каждому аргументу функции из $\mathrm{CHV}\left([-\pi, \pi]^{m}\right)$ ее ряд Фурье равномерно сходится по Прингсхейму.

C другой стороны, при $m \geqslant 3$ для любых последовательностей $\Lambda^{2}, \ldots, \Lambda^{m}$, не удовлетворяющих условию (3) (при р = 1), найдется непрерывная $2 \pi$-периодическая по каждому аргументу функиия из $\left(H, \Lambda^{2}, \ldots, \Lambda^{m}\right) \mathrm{BV}\left([-\pi, \pi]^{m}\right), \partial \Omega я$ которой ряд Фурье расходится по кубам в нуле.

Перейдем к проблеме представления интегрируемой функции на $\mathbb{R}^{m}$, принадлежащей классам ограниченной $\Lambda$-вариации, интегралом Фурье.

Рассмотрим преобразование Фурье интегрируемой в $\mathbb{R}^{m}$ функции:

$$
\hat{f}(\boldsymbol{\xi})=\frac{1}{(2 \pi)^{m / 2}} \int_{\mathbb{R}^{m}} f(\mathbf{t}) e^{-i(\boldsymbol{\xi}, \mathbf{t})} d \mathbf{t}
$$

и частичные интегралы Фурье по параллелепипедам:

$$
\begin{aligned}
\sigma_{\mathbf{A}}(f, \mathbf{x}) & =\frac{1}{(2 \pi)^{m / 2}} \int_{-A^{1}}^{A^{1}} \cdots \int_{-A^{m}}^{A^{m}} \hat{f}(\boldsymbol{\xi}) e^{i(\mathbf{x}, \boldsymbol{\xi})} d \boldsymbol{\xi} \\
& =\frac{1}{\pi^{m}} \int_{\mathbb{R}^{m}} f(\mathbf{x}+\mathbf{t}) \prod_{j=1}^{m} \frac{\sin A^{j} t^{j}}{t^{j}} d \mathbf{t} .
\end{aligned}
$$

В одномерном случае из теоремы А и принципа равносходимости (см., например, $[6$, гл. 16, п. 1]) следует

Teорема A'. Пусть $f \in \operatorname{HBV}(\mathbb{R}) \cap L(\mathbb{R})$. Тогда в каждой точке $\mathbb{R}$ интеграл Фурье функиии $f$ сходится в смысле главного значения к величине $\frac{1}{2}(f(x+0)+$ $f(x-0))$ и сходимость равномерна на любом отрезке, лежащем внутри интервала непрерьвности функции. Если $\mathrm{HBV}(\mathbb{R}) \subset \Lambda \mathrm{BV}(\mathbb{R})$ - собственное подмножество, то найдется функиия $f \in \Lambda \mathrm{BV}(\mathbb{R}) \cap L(\mathbb{R})$, интеграл Фурье которой расходится в некоторой точке (в смысле главного значения).

В многомерном случае свойство равносходимости, вообще говоря, не выполняется, поэтому вопрос о представимости функции интегралом Фурье представляет самостоятельньй интерес.

Для двумерного случая автором [7] был получен следуюший результат.

Teоpema Е. Пусть $f \in L\left(\mathbb{R}^{2}\right) \cap \Lambda \mathrm{BV}\left(\mathbb{R}^{2}\right)$, где $\frac{\lambda_{n}}{n} \downarrow 0$. Тогда в каждой регулярной точке $(x, y) \in \mathbb{R}^{2}$ функции $f$ имеет место равенство

$$
\lim _{A, B \rightarrow+\infty} \sigma_{A, B}(f,(x, y))=f^{*}(x, y)
$$

Настоящая работа посвящена обобщению этой теоремы на случай произвольной размерности. Основньм результатом является следующая 
Tеорема 1. Пусть $f \in L\left(\mathbb{R}^{m}\right) \cap \operatorname{HBV}\left(\mathbb{R}^{m}\right)$. Для заданных $\delta>0, B \geqslant \delta u$ точки $\mathbf{x}$ положим

$$
E_{\delta, B}(\mathbf{x})=\left\{\mathbf{t} \in \mathbb{R}^{m}|\exists j:| x^{j}-t^{j}|\leqslant \delta, \exists k:| x^{k}-t^{k} \mid \geqslant B\right\} .
$$

Тогда в каждой регулярной точке $\mathbf{x} \in \mathbb{R}^{m}$ функиии $f$, для которой выполнены два условия:

(A) $\lim _{\|\boldsymbol{\delta}\| \rightarrow+0} \sum_{\boldsymbol{\sigma} \in\{-1,1\} m} V_{H}(f ;(\mathbf{x}, \mathbf{x}+\boldsymbol{\sigma} \boldsymbol{\delta}))=0$

(Б) найдутся $\delta_{0}>0$ u $B \geqslant \delta_{0}$ maкие, что $f \in \mathrm{CHV}(\Delta)$ для любого параллелепипеда $\Delta \subset E_{\delta_{0}, B}(\mathbf{x})$,

имеет место равенство

$$
\lim _{\mathbf{A} \rightarrow+\infty} \sigma_{\mathbf{A}}(f, \mathbf{x})=f^{*}(\mathbf{x}) .
$$

Здесь $\mathbf{A} \rightarrow+\infty$ означает, что $\min _{j} A^{j} \rightarrow+\infty$, m. е. сходимость понимается в смисле Прингсхейма.

В частности, справедливо следуюшее утверждение, которое непосредственно вытекает из теоремы 1 и леммы 3 (см. $\S 2)$.

СлЕДСТВИЕ 1. Пусть $f \in L\left(\mathbb{R}^{m}\right) \cap \mathrm{CHV}\left(\mathbb{R}^{m}\right)$. Тогда в каждой регулярной точке $\mathbf{x} \in \mathbb{R}^{m}$ функиии $f$ имеет место равенство

$$
\lim _{\mathbf{A} \rightarrow+\infty} \sigma_{\mathbf{A}}(f, \mathbf{x})=f^{*}(\mathbf{x}) .
$$

Используя теорему 1, покажем, что в теореме С условие непрерьвности несущественно. Точнее, справедлива

TеOрема 2. Пусть $f \in \operatorname{HBV}\left([-\pi, \pi]^{m}\right)$. Тогда в кажсдой регулярной точке $\mathbf{x} \in[-\pi, \pi]^{m}$ функиии $f$, для которой

$$
\lim _{\|\boldsymbol{\delta}\| \rightarrow+0} \sum_{\boldsymbol{\sigma} \in\{-1,1\}^{m}} V_{H}(f ;(\mathbf{x}, \mathbf{x}+\boldsymbol{\sigma} \boldsymbol{\delta}))=0,
$$

ее ряд Фурье сходится по Прингсхейму к величине $f^{*}(\mathbf{x})$.

Отсюда также вытекает (с учетом леммы 3 ) следствие, усиливающее теорему D.

СледСТВИЕ 2. Пусть $f \in \mathrm{CHV}\left([-\pi, \pi]^{m}\right)$. Тогда в каждой регулярной точ$\kappa е \mathbf{x} \in[-\pi, \pi]^{m}$ функиии $f$ ее ряд Фурье сходится по Прингсхейму $к$ величине $f^{*}(\mathbf{x})$.

Отметим, что автором [5] было доказано следующее утверждение.

ТЕОРема F. Для любой последовательности $\Lambda \in \mathbb{L}$ справедливо равенство

$$
\mathrm{C} \Lambda \mathrm{V}(\Delta)=\bigcup_{M \in \mathbb{L}: \frac{\mu_{n}}{\lambda_{n}} \downarrow 0} \operatorname{MBV}(\Delta)
$$

Поэтому при $m=2$ теорема Е и следствие 1 равносильны. Но поскольку О. С. Драгошанским доказано [8], что классы $\mathrm{CHV}\left(\mathbb{R}^{2}\right)$ и $\mathrm{HBV}\left(\mathbb{R}^{2}\right)$ совпадают, то получаем 
СлЕДСТвИЕ 3. Пусть $f \in L\left(\mathbb{R}^{2}\right) \cap \mathrm{HBV}\left(\mathbb{R}^{2}\right)$. Тогда в каждой регулярной точке $(x, y) \in \mathbb{R}^{2}$ функции $f$ имеет место равенство

$$
\lim _{A, B \rightarrow+\infty} \sigma_{A, B}(f,(x, y))=f^{*}(x, y)
$$

В $\S 5$ настоящей работы построены примеры, показывающие, что в теореме 1 нельзя, вообще говоря, исключить ни условие (А), ни условие (Б). В частности, в отличие от двумерного случая, при $m \geqslant 3$ распространить теорему 1 на весь класс $\operatorname{HBV}\left(\mathbb{R}^{m}\right) \cap L\left(\mathbb{R}^{m}\right)$ нельзя.

Приведем точные формулировки доказанных в $\S 5$ утверждений.

ТЕОРема 3. Пусть $m \geqslant 3$ и последовательности $\Lambda^{2}, \ldots, \Lambda^{m} \in \mathbb{L}$ таковы, чmo

$$
\sum_{k=1}^{\infty} \frac{1}{\lambda_{k}^{2} \cdots \lambda_{k}^{m}}<\infty
$$

Тогда в классе $\left(H, \Lambda^{2}, \ldots, \Lambda^{m}\right) \mathrm{BV}\left(\mathbb{R}^{m}\right)$ существует непрерывная функция $f(\mathbf{x}) \in L\left(\mathbb{R}^{m}\right)$, тождественно равная нулювне $(-\pi, \pi)^{m}$, такая, что числовая последовательность $\sigma_{\mathbf{A}}(f, 0)$ расходится даже в смысле сходимости по кубам.

ТЕОРема 4. Пусть $m \geqslant 3$ и последовательности $\Lambda^{2}, \ldots, \Lambda^{m} \in \mathbb{L}$ удовлетворяют условию (4). Тогда в классе $\left(H, \Lambda^{2}, \ldots, \Lambda^{m}\right) \mathrm{BV}\left(\mathbb{R}^{m}\right)$ существует непрерывная функиия $f(\mathbf{x}) \in L\left(\mathbb{R}^{m}\right)$, тождественно равная нулю на $(-\pi, \pi)^{m}$, такая, что числовая последовательность $\sigma_{\mathbf{A}}(f, 0)$ расходится даже в смицсле сходимости по кубам.

\section{§. Необходимые свойства $\Lambda$-вариации}

Лемма 1. Ecлu $f \in\left(\Lambda^{1}, \ldots, \Lambda^{m}\right) \operatorname{BV}(\Delta)$, əдe $\Delta \subset \mathbb{R}^{m}, u \inf _{\Delta}|f(\mathbf{x})|=0, m o$

$$
\sup _{\Delta}|f(\mathbf{x})| \leqslant\left(\sum_{j=1}^{m} \lambda_{1}^{j}\right) V_{\Lambda^{1}, \ldots, \Lambda^{m}}(f ; \Delta) .
$$

ДокАЗАТЕльСтво. Достаточно доказать, что для любых точек $\mathbf{x}_{1}$ и $\mathbf{x}_{2}$ выполнена оценка

$$
\left|f\left(\mathbf{x}_{1}\right)-f\left(\mathbf{x}_{2}\right)\right| \leqslant\left(\sum_{j=1}^{m} \lambda_{1}^{j}\right) V_{\Lambda}(f ; \Delta) .
$$

Это неравенство следует из того, что если точки $\mathbf{x}_{1}$ и $\mathbf{x}_{2}$ различаются лишь по $j$-й координате, то

$$
\left|f\left(\mathbf{x}_{1}\right)-f\left(\mathbf{x}_{2}\right)\right|=\lambda_{1}^{j} \frac{\left|f\left(\mathbf{x}_{1}\right)-f\left(\mathbf{x}_{2}\right)\right|}{\lambda_{1}^{j}} \leqslant \lambda_{1}^{j} V_{\Lambda^{j}}^{x^{j}}(f ; \Delta) .
$$

Лемма доказана. 
Лемма 2. $E c л u f \in \Lambda \mathrm{BV}(\Delta), g \in \Lambda \mathrm{BV}\left(\Delta^{j}\right)$, mo $F(\mathbf{x})=f(\mathbf{x}) g\left(x^{j}\right) \in \Lambda \mathrm{BV}(\Delta)$ и справедлива оценка

$$
V_{\Lambda}(F ; \Delta) \leqslant C(m)\left(V_{\Lambda}(f ; \Delta) V_{\Lambda}\left(g ; \Delta^{j}\right)+V_{\Lambda}(f ; \Delta) \sup _{\Delta^{j}}|g|+V_{\Lambda}\left(g ; \Delta^{j}\right) \sup _{\Delta}|f|\right) .
$$

ДоКАЗАТЕЛЬСТВо. Без ограничения обшности предположим, что $j=1$. Если $1 \notin \alpha$, то

$$
V_{\Lambda}^{x^{\alpha}}(F ; \Delta) \leqslant V_{\Lambda}^{x^{\alpha}}(f ; \Delta) \sup _{\Delta^{1}}|g| .
$$

Пусть $\alpha=\{1, \ldots, p\}, \beta=\{p+1, \ldots, m\}$; тогда для любого $x^{\beta} \in \Delta^{\beta}$ вьполнено равенство

$$
F\left(I_{k^{\alpha}}^{\alpha}, x^{\beta}\right)=f\left(I_{k^{\alpha}}^{\alpha}, x^{\beta}\right) g\left(b_{k^{1}}^{1}\right)+f\left(a_{k^{1}}^{1}, I_{k^{2}}^{2} \times \cdots \times I_{k^{p}}^{p}, x^{\beta}\right) g\left(I_{k^{1}}^{1}\right) .
$$

Отсюда при $p>1$ следует оценка

$$
V_{\Lambda}^{x^{\alpha}}(F ; \Delta) \leqslant V_{\Lambda}(f ; \Delta) \sup _{\Delta^{1}}|g|+V_{\Lambda}(f ; \Delta) V_{\Lambda}\left(g ; \Delta^{j}\right),
$$

а при $p=1$ - оценка

$$
V_{\Lambda}^{x^{1}}(F ; \Delta) \leqslant V_{\Lambda}(f ; \Delta) \sup _{\Delta^{1}}|g|+V_{\Lambda}\left(g ; \Delta^{j}\right) \sup _{\Delta}|f|
$$

Лемма доказана.

ЛЕмма 3. Если функиия $f(x)$ принадлежит классу $\mathrm{CHV}\left(\mathbb{R}^{m}\right)$ и в точке $\mathbf{x}_{0}$ существует предел $f\left(\mathbf{x}_{0}+0\right)$, то

$$
\lim _{\delta \rightarrow+0} V_{H}\left(f ; \bigotimes_{j=1}^{m}\left(x_{0}^{j}, x_{0}^{j}+\delta\right)\right)=0
$$

Лемма доказана в работе автора [5, теорема 2].

Будем также использовать следуюшие простейшие свойства $\Lambda$-вариации:

$$
V_{\Lambda^{1}, \ldots, \Lambda^{m}}(f+g ; \Delta) \leqslant V_{\Lambda^{1}, \ldots, \Lambda^{m}}(f ; \Delta)+V_{\Lambda^{1}, \ldots, \Lambda^{m}}(g ; \Delta) ;
$$

если $\Delta_{1}$ и $\Delta_{2}$ - два параллелепипеда, образующие в объединении параллелепипед и пересекаюшиеся по общей грани, то

$$
V_{\Lambda^{1}, \ldots, \Lambda^{m}}\left(f ; \Delta_{1} \cup \Delta_{2}\right) \leqslant V_{\Lambda^{1}, \ldots, \Lambda^{m}}\left(f ; \Delta_{1}\right)+V_{\Lambda^{1}, \ldots, \Lambda^{m}}\left(f ; \Delta_{2}\right) .
$$




\section{§ 3. Вспомогательные оценки}

Лемма 4. Пусть $f \in\left(\Lambda^{1}, \ldots, \Lambda^{m}\right) \mathrm{BV}\left(\mathbb{R}^{m}\right)$, где $\lambda_{n}^{j} / n \leqslant C\left(\Lambda^{j}\right),\{1, \ldots, m\}=$ $\alpha \sqcup \beta$, где $\alpha$ непусто, и $\Delta^{\alpha} \subset \mathbb{R}^{|\alpha|}$ - произвольньй параллелепипед, $\Delta^{\beta} \subset \mathbb{R}^{|\beta|}$ ограниченный параллелепипед. Определим функиию $\varphi_{\mathbf{A}}\left(t^{\alpha}\right)$ формулой

$$
\varphi_{\mathbf{A}}\left(t^{\alpha}\right)=\int_{\Delta^{\beta}} f\left(x^{\alpha}+t^{\alpha}, x^{\beta}+t^{\beta}\right) \prod_{j \in \beta} \frac{\sin A^{j} t^{j}}{t^{j}} d t^{\beta} .
$$

Тогда для любых $A^{j}>1$ выполнена оценка

$$
V_{\Lambda^{\alpha}}\left(\varphi_{\mathbf{A}} ; \Delta^{\alpha}\right) \leqslant C\left(m, \Lambda^{\beta}\right) V_{\Lambda^{1}, \ldots, \Lambda^{m}}\left(f ;\left(x^{\alpha}+\Delta^{\alpha}\right) \times\left(x^{\beta}+\Delta^{\beta}\right)\right) .
$$

Лемма 5. Пусть $g \in \operatorname{HBV}(\Delta), \Delta=\bigotimes_{j=1}^{m}\left[a^{j}, b^{j}\right]-$ конечный или бесконечный промежуток в $\mathbb{R}^{m}$ (во втором случае дополнительно потребуем, чтобы $g$ была интегрируема по Лебегу на $\Delta)$. Тогда существует величина $C(m)$ такая, что для любьх $A^{j}>1$ и для любого $k$ при $\zeta=\left\lfloor\frac{1}{2 \pi} \min _{t \in\left[a^{k}, b^{k}\right]}\left|A^{k} t\right|\right\rfloor$ (здесь и далее $\lfloor x\rfloor-$ челая часть числа $x$ ) выполнена оценка

$$
\begin{aligned}
\left|\int_{\Delta} g(\mathbf{t}) \prod_{j=1}^{m} \frac{\sin A^{j} t^{j}}{t^{j}} d \mathbf{t}\right| \leqslant C(m) & \left(V_{H, \ldots, H, H_{2 \zeta}^{k}, H, \ldots, H}(g ; \Delta)\right. \\
& \left.+\frac{1}{1+\zeta}\left(\sup _{\Delta}|g|+V_{H}(g ; \Delta)\right)\right) .
\end{aligned}
$$

Здесь верхний индекс $k$ при $H_{2 \zeta}$ показывает, что сдвигается $k$-я последовательность.

ДоКАЗАТЕЛЬСТво ЛЕмм 4 и 5. Проведем параллельно доказательство этих лемм по индукции. Прежде всего, отметим, что утверждение леммы 5 достаточно доказать для случая ограниченного промежутка. Действительно, если

$$
V_{H, \ldots, H, H_{2 \zeta}^{k}, H, \ldots, H}(g ; \Delta)+\frac{1}{1+\zeta}\left(\sup _{\Delta}|g|+V_{H}(g ; \Delta)\right)=0
$$

то это означает, что $g \equiv 0$ на $\Delta$, т.е. утверждение леммы заведомо выполнено; в противном случае в силу свойств интеграла Лебега можно подобрать конечньй параллелепипед $\Delta^{\prime} \subset \Delta$ так, чтобы

$$
\left|\int_{\Delta \backslash \Delta^{\prime}} g(\mathbf{t}) \prod_{j=1}^{m} \frac{\sin A^{j} t^{j}}{t^{j}} d \mathbf{t}\right| \leqslant\left(V_{H, \ldots, H, H_{2 \zeta}^{k}, H, \ldots, H}(g ; \Delta)+\frac{1}{1+\zeta} \sup _{\Delta}|g|\right) .
$$

Утверждение леммы 4 при $m=1$ тривиально, так как непустота $\alpha$ влечет пустоту $\beta$, т.е. в этом случае

$$
\varphi_{A}(t)=f(x+t) .
$$

Докажем лемму 5 при $m=1$. (Отметим, что для $\zeta=0$ доказательство фактически содержится в [9].) 
Пусть $\Delta=(a, b)$. Достаточно рассмотреть случай $a, b \geqslant 0$ (в противном случае разобьем интервал $(a, b)$ на две части). Если $b-a \leqslant \frac{4 \pi}{A}$, то справедлива оценка

$$
\left|\int_{a}^{b} g(t) \frac{\sin A t}{t} d t\right|=\left|\int_{A a}^{A b} g\left(\frac{s}{A}\right) \frac{\sin s}{s} d s\right| \leqslant \frac{C}{A a+1} \sup _{\Delta}|g(t)|
$$

Пусть теперь $b-a>\frac{4 \pi}{A}$. Выберем натуральные $K \geqslant K^{\prime}$ так, чтобы

$$
\frac{\left(2 K^{\prime}-3\right) \pi}{A} \leqslant a<\frac{\left(2 K^{\prime}-1\right) \pi}{A}<\frac{(2 K+1) \pi}{A} \leqslant b<\frac{(2 K+3) \pi}{A} .
$$

Тогда $K^{\prime}=\left\lfloor\frac{a A}{2 \pi}+\frac{3}{2}\right\rfloor$. Отметим, что при этом $K^{\prime}=\zeta+1$ или $K^{\prime}=\zeta+2$. Имеем

$$
\begin{aligned}
\int_{a}^{b} g(t) \frac{\sin A t}{t} d t= & \int_{a}^{\left(2 K^{\prime}-1\right) \pi / A} g(t) \frac{\sin A t}{t} d t+\int_{\left(2 K^{\prime}-1\right) \pi / A}^{(2 K+1) \pi / A} g(t) \frac{\sin A t}{t} d t \\
& +\int_{(2 K+1) \pi / A}^{b} g(t) \frac{\sin A t}{t} d t .
\end{aligned}
$$

Первое и третье слагаемые оцениваются так же, как в (7). Оценим второе слагаемое. Имеем

$$
\begin{aligned}
J_{2} & =\int_{\left(2 K^{\prime}-1\right) \pi / A}^{(2 K+1) \pi / A} g(t) \frac{\sin A t}{t} d t=\sum_{j=2 K^{\prime}-1}^{2 K} \int_{j \pi / A}^{(j+1) \pi / A} g(t) \frac{\sin A t}{t} d t \\
& =\sum_{j=2 K^{\prime}-1}^{2 K} \int_{j \pi}^{(j+1) \pi} g\left(\frac{s}{A}\right) \frac{\sin s}{s} d s=\sum_{j=2 K^{\prime}-1}^{2 K} \int_{0}^{\pi} g\left(\frac{s+j \pi}{A}\right) \frac{\sin (s+j \pi)}{s+j \pi} d s \\
& =\sum_{j=2 K^{\prime}-1}^{2 K}(-1)^{j} \int_{0}^{\pi} g\left(\frac{s+j \pi}{A}\right) \frac{\sin s}{s+j \pi} d s .
\end{aligned}
$$

Группируя слагаемые по два, получим

$$
\begin{aligned}
J_{2}= & \sum_{j=K^{\prime}}^{K} \int_{0}^{\pi}\left(\frac{g((t+2 j \pi) / A)}{t+2 j \pi}-\frac{g((t+(2 j-1) \pi) / A)}{t+(2 j-1) \pi}\right) \sin t d t \\
= & \frac{1}{\pi} \int_{0}^{\pi} \sum_{j=K^{\prime}}^{K} \frac{g((t+2 j \pi) / A)-g((t+(2 j-1) \pi) / A)}{t / \pi+(2 j-1)} \sin t d t \\
& +\sum_{j=K^{\prime}}^{K} \int_{0}^{\pi} g\left(\frac{t+2 j \pi}{A}\right)\left(\frac{1}{t+2 j \pi}-\frac{1}{t+(2 j-1) \pi}\right) \sin t d t=J_{2}^{\prime}+J_{2}^{\prime \prime} .
\end{aligned}
$$


Видно, что подьнтегральное выражение в $J_{2}^{\prime}$ не превосходит по модулю $H_{2 K^{\prime}-2^{-в а-~}}$ риации функции $g$, поэтому $\left|J_{2}^{\prime}\right| \leqslant V_{H_{2 \zeta}}(g ; \Delta)$. Для второй суммы имеем оценку

$$
\begin{aligned}
\left|J_{2}^{\prime \prime}\right| & \leqslant \sup _{\Delta}|g| \sum_{j=K^{\prime}}^{K} \int_{0}^{\pi} \frac{\pi}{(t+2 j \pi)(t+(2 j-1) \pi)} d t \\
& \leqslant \sup _{\Delta}|g| \sum_{j=K^{\prime}}^{\infty} \frac{1}{(2 j-1)^{2}} \leqslant \frac{C}{\zeta+1} \sup _{\Delta}|g| .
\end{aligned}
$$

Таким образом, лемма 5 для одномерного случая доказана.

Пусть для $(m-1)$-мерного случая утверждения обеих лемм уже установлены.

Докажем лемму 4 для $m$-мерного случая. Из соображений симметрии достаточно рассмотреть случай $\alpha=\{1, \ldots, p\}$, где $1 \leqslant p \leqslant m-1$. Отметим, что оценка младших компонент вариации функции $\varphi_{\text {A }}$ (т.е. ее вариации не по всем переменным $\mathbf{x}^{\alpha}$ ) следует из предположения индукции.

Пусть $F(\mathbf{t})=f(\mathbf{x}+\mathbf{t})$. Рассмотрим системы интервалов $\left\{I_{k^{j}}^{j}\right\} \in \Omega\left(\Delta^{j}\right), j=$ $1, \ldots, p$. Если $\varepsilon_{k^{\alpha}}=\operatorname{sign}\left(\varphi_{\mathbf{A}}\left(I_{k^{\alpha}}^{\alpha}\right)\right)$, то

$$
Z=\sum_{k^{\alpha}} \frac{\left|\varphi_{\mathbf{A}}\left(I_{k^{\alpha}}^{\alpha}\right)\right|}{\lambda_{k^{\alpha}}^{\alpha}}=\sum_{k^{\alpha}} \frac{\varepsilon_{k^{\alpha}} \varphi_{\mathbf{A}}\left(I_{k^{\alpha}}^{\alpha}\right)}{\lambda_{k^{\alpha}}^{\alpha}}=\int_{\Delta^{\beta}} \psi\left(t^{\beta}\right) \prod_{j \in \beta} \frac{\sin A^{j} t^{j}}{t^{j}} d t^{\beta},
$$

где $\psi$ определена формулой

$$
\psi\left(t^{\beta}\right)=\sum_{k^{\alpha}} \frac{\varepsilon_{k^{\alpha}}}{\lambda_{k^{\alpha}}^{\alpha}} F\left(I_{k^{\alpha}}^{\alpha}, t^{\beta}\right)
$$

Заметим, что в силу определения $\Lambda$-вариации

$$
\sup _{\Delta^{\beta}}|\psi| \leqslant V_{\Lambda^{1}, \ldots, \Lambda^{m}}(F ; \Delta)
$$

Оценим $\left(\Lambda^{p+1}, \ldots, \Lambda^{m}\right)$-вариацию $\psi$. Рассмотрим непустое $\gamma \subset \beta$ и $\eta=\beta \backslash \gamma$. Для любых систем интервалов $\left\{I_{k^{j}}^{j}\right\} \in \Omega\left(\Delta^{j}\right), j \in \gamma$, выполнено

$$
\sum_{k^{\gamma}} \frac{\left|\psi\left(I_{k^{\gamma}}^{\gamma}\right)\right|}{\lambda_{k^{\gamma}}^{\gamma}} \leqslant \sum_{k^{\alpha}, k^{\gamma}} \frac{\left|F\left(I_{k^{\alpha}}^{\alpha} \times I_{k^{\gamma}}^{\gamma}, x^{\eta}\right)\right|}{\lambda_{k^{\alpha}}^{\alpha} \lambda_{k^{\gamma}}^{\gamma}} \leqslant V_{\Lambda^{\alpha}, \Lambda^{\gamma}}^{x^{\alpha}, x^{\gamma}}(F ; \Delta) .
$$

Отсюда, переходя в левой части неравенства к верхней грани по $\Omega\left(\Delta^{j}\right), j \in \gamma$, и по $x \in \Delta^{\eta}$, а затем суммируя по $\gamma$, получаем

$$
V_{\Lambda^{\beta}}\left(\psi ; \Delta^{\beta}\right) \leqslant C(m) V_{\Lambda}(F ; \Delta) .
$$

Из соотношений (8) по лемме 5 (в силу непустоты $\alpha$ выполнено $|\beta|<m$ ) получаем

$$
Z \leqslant C(m-p)\left(V_{H}\left(\psi ; \Delta^{\beta}\right)+\sup _{\Delta^{\beta}}|\psi|\right) \leqslant C\left(m, \Lambda^{\beta}\right)\left(V_{\Lambda^{\beta}}\left(\psi ; \Delta^{\beta}\right)+\sup _{\Delta^{\beta}}|\psi|\right) .
$$


Здесь важно отметить, что константа в последнем неравенстве определяется величиной $\sup _{j \in \beta} \sup _{n} \frac{\lambda_{n}^{j}}{n}$, т. е. не зависит от $\Lambda^{\alpha}$. Переходя к верхней грани по $\Omega\left(\Delta^{j}\right)$, $j \in \alpha$, получаем оценку

$$
V_{\Lambda}\left(\varphi ; \Delta^{\alpha}\right) \leqslant C\left(m, \Lambda^{\beta}\right) V_{\Lambda}(F ; \Delta)=C\left(m, \Lambda^{\beta}\right) V_{\Lambda}(f ; \mathbf{x}+\Delta)
$$

Тем самым лемма 4 доказана для $m$-мерного случая.

Пусть теперь лемма 4 доказана для $m$-мерного случая, а лемма 5 - для $(m-1)$ мерного случая. Докажем лемму 5 для $m$-мерного случая.

Без ограничения общности предположим, что $k=1$. Пусть $t^{*}=\left(t^{2}, \ldots, t^{m}\right)$ и $\Delta^{*}=\bigotimes_{j=2}^{m}\left[a^{j}, b^{j}\right]$. Имеем

$$
Z_{1}=\int_{\Delta} g(\mathbf{t}) \prod_{j=1}^{m} \frac{\sin A^{j} t^{j}}{t^{j}} d \mathbf{t}=\int_{a^{1}}^{b^{1}} \frac{\sin A^{1} t^{1}}{t^{1}}\left(\int_{\Delta^{*}} g\left(t^{1}, t^{*}\right) \prod_{j=2}^{m} \frac{\sin A^{j} t^{j}}{t^{j}} d t^{*}\right) d t^{1} .
$$

Рассмотрим функцию

$$
G\left(t^{1}\right)=\int_{\Delta^{*}} g\left(t^{1}, t^{*}\right) \prod_{j=2}^{m} \frac{\sin A^{j} t^{j}}{t^{j}} d t^{*}
$$

По лемме 4 имеем $G\left(t^{1}\right) \in \operatorname{HBV}\left(\Delta^{1}\right)$ и $V_{H_{\zeta}}\left(G ; \Delta^{1}\right) \leqslant C_{1}(m) V_{H_{\zeta}, H, \ldots, H}(g ; \Delta)$. По предположению индукции $\sup _{\Delta^{1}}|G| \leqslant C(m-1)\left(V_{H}(g ; \Delta)+\sup _{\Delta}|g|\right)$. В силу основания индукции

$$
\left|Z_{1}\right| \leqslant C(1)\left(V_{H_{\zeta}}\left(G ; \Delta^{1}\right)+\frac{1}{\zeta+1} \sup _{\Delta^{1}}|G|\right)
$$

Комбинируя последние неравенства, получим

$$
\left|Z_{1}\right| \leqslant C(m)\left(V_{H_{\zeta}, H, \ldots, H}(g ; \Delta)+\frac{1}{\zeta+1}\left(\sup _{\Delta}|g|+V_{H}(g ; \Delta)\right)\right) .
$$

Леммы 4 и 5 доказаны.

ЛЕмма 6. Пусть $\Delta=\bigotimes_{j=1}^{m} \Delta^{j}$, где $\Delta^{j}=\left[a^{j}, b^{j}\right]$. Пусть $f \in \Lambda \mathrm{BV}(\Delta)$. Тогда для $\varkappa^{j}=\left(b^{j}-a^{j}\right) / \pi$ справедлива оченка

$$
\left|\int_{\Delta} f(\mathbf{t}) \prod_{j=1}^{m} \sin A^{j} t^{j} d \mathbf{t}\right| \leqslant C(\Delta)\left(\frac{V_{\Lambda}(f ; \Delta)}{\prod_{j=1}^{m} \Lambda\left(\left\lfloor\varkappa^{j} A^{j}\right\rfloor-1\right)}+\sup _{\Delta}|f|\left(\max _{j} \frac{1}{A^{j}}\right)\right) .
$$

Отметим, что при $m=1$ доказательство лишь несушественно отличается от доказательства аналогичной оценки для коэффициентов Фурье (см. [10]). 
ДокАЗАТЕльСТво. Обозначим $\Delta^{(\mathbf{A})}=\bigotimes_{j=1}^{m}\left[a^{j}, b^{j}-\frac{\pi}{A^{j}}\right]$. Введем обозначение для смешанного приращения:

$$
f\left(\mathbf{t}, \mathbf{t}+\frac{\pi}{\mathbf{A}}\right)=f\left(\bigotimes_{j=1}^{m}\left(t^{j}, t^{j}+\frac{\pi}{A^{j}}\right)\right) .
$$

Сначала докажем индукцией по $m$, что

$$
\int_{\Delta} f(\mathbf{t}) \prod_{j=1}^{m} \sin A^{j} t^{j} d \mathbf{t}=\frac{(-1)^{m}}{2^{m}} \int_{\Delta(\mathbf{A})} f\left(\mathbf{t}, \mathbf{t}+\frac{\pi}{\mathbf{A}}\right) \prod_{j=1}^{m} \sin A^{j} t^{j} d \mathbf{t}+R(f, \Delta, \mathbf{A})
$$

где

$$
|R(f, \Delta, \mathbf{A})| \leqslant C(\Delta) \sup _{\Delta}|f|\left(\max _{j} \frac{1}{A^{j}}\right) .
$$

При $m=1$ имеем: если $|b-a| \leqslant \pi / A$, то $\Delta^{(\mathbf{A})}$ пусто и нетрудно видеть, что интеграл, стояший в левой части (9), не превосходит по модулю правой части (10); в противном случае из равенства

$$
J=\int_{a}^{b} f(t) \sin A t d t=-\int_{a-\frac{\pi}{A}}^{b-\frac{\pi}{A}} f\left(t+\frac{\pi}{A}\right) \sin A t d t
$$

получаем

$$
\begin{aligned}
J= & -\frac{1}{2} \int_{a}^{b-\frac{\pi}{A}}\left(f\left(t+\frac{\pi}{A}\right)-f(t)\right) \sin A t d t+\frac{1}{2} \int_{b-\frac{\pi}{A}}^{b} f(t) \sin A t d t \\
& -\frac{1}{2} \int_{a-\frac{\pi}{A}}^{a} f\left(t+\frac{\pi}{A}\right) \sin A t d t .
\end{aligned}
$$

Отсюда видно, что в данном случае соотношение (9) справедливо.

Пусть для $(m-1)$-мерного случая равенство (9) установлено. Положим $\left|\Delta^{j}\right|=$ $b^{j}-a^{j}$. Если для некоторого $k$ выполнено $\left|\Delta^{k}\right| \leqslant \pi / A^{k}$, то $\Delta^{(\mathbf{A})}$ пусто и

$$
\left|\int_{\Delta} f(\mathbf{t}) \prod_{j=1}^{m} \sin A^{j} t^{j} d \mathbf{t}\right| \leqslant \frac{\pi}{A^{k}} \sup _{\Delta}|f(\mathbf{t})| \prod_{j \neq k}\left|\Delta^{j}\right|
$$

т. е. равенство (9) справедливо.

Пусть теперь для любого $j$ выполнено неравенство $\left|\Delta^{j}\right|>\pi / A^{j}$. Положим $\Delta^{*}=\bigotimes_{j=2}^{m} \Delta^{j}$ и $t^{*}=\left(t^{2}, \ldots, t^{m}\right)$. Тогда имеем

$$
\begin{aligned}
J & =\int_{\Delta} f(\mathbf{t}) \prod_{j=1}^{m} \sin A^{j} t^{j} d \mathbf{t} \\
& =-\int_{a^{1}-\frac{\pi}{A^{1}}}^{b^{1}-\frac{\pi}{A^{1}}} \int_{\Delta^{*}} f\left(t^{1}+\frac{\pi}{A^{1}}, t^{*}\right) \prod_{j=1}^{m} \sin A^{j} t^{j} d t^{*} d t^{1} .
\end{aligned}
$$


Отсюда получаем

$$
\begin{aligned}
J=\frac{1}{2}\left(\int_{\Delta} f(\mathbf{t}) \prod_{j=1}^{m} \sin A^{j} t^{j} d \mathbf{t}\right. \\
\left.\quad-\int_{a^{1}-\frac{\pi}{A^{1}}}^{b^{1}-\frac{\pi}{A^{1}}} \int_{\Delta^{*}} f\left(t^{1}+\frac{\pi}{A^{1}}, t^{*}\right) \prod_{j=1}^{m} \sin A^{j} t^{j} d t^{*} d t^{1}\right)=J_{1}+J_{2}+J_{3},
\end{aligned}
$$

где

$$
\begin{aligned}
& J_{1}=\frac{1}{2} \int_{a^{1}}^{b^{1}-\frac{\pi}{A^{1}}} \int_{\Delta^{*}}\left(f(\mathbf{t})-f\left(t^{1}+\frac{\pi}{A^{1}}, t^{*}\right)\right) \prod_{j=1}^{m} \sin A^{j} t^{j} d t^{*} d t^{1}, \\
& J_{2}=\int_{b_{1}-\frac{\pi}{A^{1}}}^{b^{1}} \int_{\Delta^{*}} f(\mathbf{t}) \prod_{j=1}^{m} \sin A^{j} t^{j} d t^{*} d t^{1}, \\
& J_{3}=-\int_{a^{1}-\frac{\pi}{A^{1}}}^{a^{1}} \int_{\Delta^{*}} f\left(t^{1}-\frac{\pi}{A^{1}}, t^{*}\right) \prod_{j=1}^{m} \sin A^{j} t^{j} d t^{*} d t^{1} .
\end{aligned}
$$

Интегралы $J_{2}$ и $J_{3}$ оцениваются аналогично $(11)$. К внутреннему интегралу в $J_{1}$ применим предположение индукции. Получим, что

$$
\begin{aligned}
J_{1}= & \frac{(-1)^{m}}{2^{m}} \int_{\Delta(\mathbf{A})} f\left(\mathbf{t}, \mathbf{t}+\frac{\pi}{\mathbf{A}}\right) \prod_{j=1}^{m} \sin A^{j} t^{j} d \mathbf{t} \\
& +\frac{1}{2} \int_{a^{1}}^{b^{1}-\frac{\pi}{A^{1}}} R\left(f\left(t^{1}, \cdot\right), \Delta^{*},\left(A^{2}, \ldots, A^{m}\right)\right) d t^{1}
\end{aligned}
$$

Здесь для подынтегральной функции второго слагаемого справедлива оценка (10) из предположения индукции, а длина отрезка интегрирования не превосходит $b^{1}-a^{1}=C(\Delta)$. Таким образом, (9) установлено.

Перейдем непосредственно к доказательству утверждения леммы. Поскольку для $R(f, \Delta, \mathbf{A})$ требуемая оценка справедлива, то достаточно оценить величину

$$
\frac{1}{2^{m}} \int_{\Delta(\mathbf{A})} f\left(\mathbf{t}, \mathbf{t}+\frac{\pi}{\mathbf{A}}\right) \prod_{j=1}^{m} \sin A^{j} t^{j} d \mathbf{t}
$$

Пусть $K^{j}-$ наибольшее целое такое, что $b^{j}-a^{j}-\frac{\pi}{A^{j}} \geqslant K^{j} \frac{\pi}{A^{j}}$. Обозначим через $\Delta_{*}^{(\mathbf{A})}$ промежуток $\bigotimes_{j=1}^{m}\left[a^{j}, a^{j}+K^{j} \frac{\pi}{A^{j}}\right)$.

Заметим, что объем множества $\Delta^{(\mathbf{A})} \backslash \Delta_{*}^{(\mathbf{A})}$ не превосходит величины $\sum_{j=1}^{m} \frac{\pi}{A^{j}} \times$ $\prod_{i \neq j}\left|\Delta^{i}\right| ;$ поэтому

$$
\int_{\Delta}^{(\mathbf{A}) \backslash \Delta_{*}^{(\mathbf{A})}} f\left(\mathbf{t}, \mathbf{t}+\frac{\pi}{\mathbf{A}}\right) \prod_{j=1}^{m} \sin A^{j} t^{j} d \mathbf{t} \leqslant C(\Delta) \sup _{\Delta}|f| \max _{j} \frac{1}{A^{j}} .
$$


Рассмотрим оставшийся интеграл:

$$
\left|Z_{5}\right|=\left|\int_{\Delta_{*}^{(\mathbf{A})}} f\left(\mathbf{t}, \mathbf{t}+\frac{\pi}{\mathbf{A}}\right) \prod_{j=1}^{m} \sin A^{j} t^{j} d \mathbf{t}\right| \leqslant \int_{\Delta_{*}^{(\mathbf{A})}}\left|f\left(\mathbf{t}, \mathbf{t}+\frac{\pi}{\mathbf{A}}\right)\right| d \mathbf{t}=Z_{5}^{\prime} .
$$

Продолжим теперь функцию $f(\mathbf{t})$, определенную на параллелепипеде $\Delta_{*}^{(\mathbf{A})}$ (полуоткрытом справа по каждой координате), на все $\mathbb{R}^{m}$ с периодами $\frac{K^{j} \pi}{A^{j}}$ по переменным $t^{j}$. В силу периодичности полученной функции $f_{0}(\mathbf{t})$ имеем для любых $k^{j}=0, \ldots, K^{j}-1$ соотношение

$$
Z_{5}^{\prime}=\int_{\Delta_{*}^{(\mathbf{A})}}\left|f_{0}\left(\bigotimes_{j=1}^{m}\left(t^{j}+k^{j} \frac{\pi}{A^{j}}, t^{j}+\left(k^{j}+1\right) \frac{\pi}{A^{j}}\right)\right)\right| d \mathbf{t} .
$$

Из линейной комбинации равенств (12) получим

$$
\begin{aligned}
Z_{5}^{\prime}= & \frac{1}{\Lambda\left(K^{1}\right) \cdots \Lambda\left(K^{m}\right)} \\
& \times \int_{\Delta_{*}^{(\mathbf{A})}} \sum_{k^{1}=0}^{K^{1}-1} \cdots \sum_{k^{m}=0}^{K^{m}-1} \frac{\left|f_{0}\left(\bigotimes_{j=1}^{m}\left(t^{j}+k^{j} \frac{\pi}{A^{j}}, t^{j}+\left(k^{j}+1\right) \frac{\pi}{A^{j}}\right)\right)\right|}{\lambda_{k^{1}+1} \cdots \lambda_{k^{m}+1}} d \mathbf{t} .
\end{aligned}
$$

Отсюда с учетом (6) имеем

$$
\begin{aligned}
\left|Z_{5}\right| & \leqslant \frac{\prod_{j=1}^{m}\left|\Delta^{j}\right|}{\Lambda\left(K^{1}\right) \cdots \Lambda\left(K^{m}\right)} V_{\Lambda}\left(f_{0} ; \bigotimes_{j=1}^{m}\left(a^{j}, a^{j}+2 K^{j} \frac{\pi}{A^{j}}\right)\right) \\
& \leqslant \frac{C(\Delta) V_{\Lambda}\left(f ; \bigotimes_{j=1}^{m}\left(a^{j}, b^{j}\right)\right)}{\Lambda\left(K^{1}\right) \cdots \Lambda\left(K^{m}\right)} .
\end{aligned}
$$

Лемма доказана.

\section{§4. Доказательство теорем о сходимости}

ДОКАЗАТЕЛЬСТВО ТЕОРЕМЫ 1. Без ограничения обшности предположим, что $\mathbf{x}=0$, и рассмотрим функцию, отличную от нуля лишь при $t^{j}>0$.

Пусть задано $\varepsilon>0$. Прежде всего, в силу условия $(\mathrm{A})$ теоремы выберем $\delta>0$ так, чтобы

$$
V_{H}\left(f ;(0, \delta)^{m}\right) \leqslant \varepsilon .
$$

При этом будем считать, что $\delta<\delta_{0}$, где $\delta_{0}-$ такое же, как в условии (Б). Пусть число $B$ - из условия (Б). Для произвольного разбиения множества $\{1, \ldots, m\}$ на три непересекаюшихся множества $\alpha, \beta$ и $\gamma$ обозначим

$$
P_{\alpha, \beta, \gamma}=\bigotimes_{j=1}^{m} J_{\alpha, \beta, \gamma}^{j}, \quad J_{\alpha, \beta, \gamma}^{j}= \begin{cases}(0, \delta), & j \in \alpha \\ {[\delta, B),} & j \in \beta \\ {[B,+\infty),} & j \in \gamma\end{cases}
$$


Тогда получаем, что

$$
\begin{aligned}
\sigma_{\mathbf{A}}(f, 0) & =\sum_{\alpha \sqcup \beta \sqcup \gamma=\{1, \ldots, m\}} S_{\alpha, \beta, \gamma} \\
& =\sum_{\alpha \sqcup \beta \sqcup \gamma=\{1, \ldots, m\}} \frac{1}{\pi^{m}} \int_{P_{\alpha, \beta, \gamma}} f(\mathbf{t}) \prod_{j=1}^{m} \frac{\sin A^{j} t^{j}}{t^{j}} d \mathbf{t} .
\end{aligned}
$$

Перейдем теперь к оценке различных слагаемых в этой сумме.

1. Пусть $\alpha=\varnothing$. Тогда соответствующее слагаемое есть линейная комбинация значений преобразования Фурье функции $f(\mathbf{t})\left(\prod_{j=1}^{m} \frac{1}{t^{j}}\right) \chi_{P_{\varnothing, \beta, \gamma}}(\mathbf{t})$ в точках $\left( \pm A^{1}, \ldots, \pm A^{m}\right)$, причем коэффициенты в этой линейной комбинации не зависят от $\mathbf{A}$. Поскольку преобразование Фурье интегрируемой функции стремится к нулю с ростом $\min _{j}\left|\xi^{j}\right|$, то при достаточно больших $A^{j}$ эти слагаемые будут меньше $\varepsilon$.

2. Пусть $\alpha$ и $\gamma$ непусты. Для определенности положим $\alpha=\{1, \ldots, p\}, \beta=$ $\{p+1, \ldots, q\}, \gamma=\{q+1, \ldots, m\}$ (остальные случаи симметричны).

Для произвольных $A^{j}>1$ рассмотрим функцию

$$
\varphi_{\mathbf{A}}\left(t^{\gamma}\right)=\prod_{j=q+1}^{m} \int_{(0, \delta)^{p} \times[\delta, B)^{q-p}} f(\mathbf{t}) \prod_{j=1}^{q} \frac{\sin A^{j} t^{j}}{t^{j}} d t^{\alpha} d t^{\beta} .
$$

Тогда

$$
S_{\alpha, \beta, \gamma}=\frac{1}{\pi^{m}} \int_{[B, \infty)^{m-q}} \varphi_{\mathbf{A}}\left(t^{\gamma}\right) \prod_{j=q+1}^{m} \frac{\sin A^{j} t^{j}}{t^{j}} d t^{\gamma} .
$$

Заметим, что так как $\varphi_{\mathbf{A}}\left(t^{\gamma}\right)$ интегрируема на параллелепипеде бесконечной меры, то по лемме 1 справедлива оценка

$$
\sup _{[B, \infty)^{m-q}}\left|\varphi_{\mathbf{A}}(t)\right| \leqslant C(m) V_{H}\left(\varphi_{\mathbf{A}} ;[B, \infty)^{m-q}\right) .
$$

По лемме 5 для $\zeta=\left\lfloor\frac{A^{m} B}{2 \pi}\right\rfloor$ справедлива оценка

$$
\left|S_{\alpha, \beta, \gamma}\right| \leqslant C(m)\left(V_{H, \ldots, H, H_{2 \zeta}}\left(\varphi_{\mathbf{A}} ;[B, \infty)^{m-q}\right)+\frac{1}{\zeta+1} V_{H}\left(\varphi_{\mathbf{A}} ;[B, \infty)^{m-q}\right)\right) .
$$

Применяя лемму 4, получаем

$$
\left|S_{\alpha, \beta, \gamma}\right| \leqslant C(m)\left(V_{H, \ldots, H, H_{2 \zeta}}\left(f ; P_{\alpha, \beta, \gamma}\right)+\frac{1}{\zeta+1} V_{H}\left(f ; P_{\alpha, \beta, \gamma}\right)\right) .
$$

При увеличении $\zeta$, т. е. при увеличении $A^{m}$, правая часть этого неравенства в силу условия (Б) теоремы стремится к нулю. Выберем $A^{m}$ столь большим, чтобы она была не больше $\varepsilon$.

3. Пусть $\gamma$ пусто, а $\alpha$ и $\beta$ непусты. Для определенности положим $\alpha=\{1, \ldots, p\}$, $\beta=\{p+1, \ldots, m\}$. Тогда рассмотрим на $[\delta, B)^{m-p}$ функции

$$
\begin{aligned}
& \varphi_{\mathbf{A}}\left(t^{\beta}\right)=\int_{(0, \delta)^{p}} f(\mathbf{t}) \prod_{j=1}^{p} \frac{\sin A^{j} t^{j}}{t^{j}} d t^{\alpha}, \\
& \psi_{\mathbf{A}}\left(t^{\beta}\right)=\prod_{j=p+1}^{m} \frac{1}{t^{j}} \int_{(0, \delta)^{p}} f(\mathbf{t}) \prod_{j=1}^{p} \frac{\sin A^{j} t^{j}}{t^{j}} d t^{\alpha} .
\end{aligned}
$$


По лемме 4 справедлива оценка

$$
V_{H}\left(\varphi_{\mathbf{A}} ;[\delta, B)^{m-p}\right) \leqslant C(m) V_{H}\left(f ; \mathbb{R}^{m}\right),
$$

из которой по лемме 2 с учетом леммы 1 вытекает оценка

$$
\left.V_{H}\left(\psi_{\mathbf{A}} ; \delta, B\right)^{m-p}\right) \leqslant \frac{C(m)}{\delta^{m-p}}\left(V_{H}\left(f ; \mathbb{R}^{m}\right)+\sup _{\mathbb{R}^{m}}|f|\right) \leqslant \frac{C(m)}{\delta^{m-p}} V_{H}\left(f ; \mathbb{R}^{m}\right) .
$$

С другой стороны, по лемме 5 с учетом леммы 1 имеем

$$
\sup _{[\delta, B)^{m-p}}\left|\psi_{\mathbf{A}}\right| \leqslant \frac{1}{\delta^{m-p}} \sup _{[\delta, B)^{m-p}}\left|\varphi_{\mathbf{A}}\right| \leqslant \frac{C(m)}{\delta^{m-p}} V_{H}\left(f ; \mathbb{R}^{m}\right) .
$$

Поскольку

$$
S_{\alpha, \beta, \varnothing}=\int_{[\delta, B)^{m-p}} \psi_{\mathbf{A}}\left(t^{\beta}\right) \prod_{j=p+1}^{m} \sin A^{j} t^{j} d t^{\beta},
$$

то по лемме 6 найдутся положительные $\varkappa^{j}$, зависящие лишь от $\delta$ и $B$, такие, что

$$
\left|S_{\alpha, \beta, \varnothing}\right| \leqslant C(\delta, B)\left(\frac{V_{H}\left(\psi_{\mathbf{A}} ;[\delta, B)^{m-p}\right)}{\prod_{j=p+1}^{m} \Lambda\left(\left\lfloor\varkappa^{j} A^{j}\right\rfloor-1\right)}+\sup _{[\delta, B)^{m-p}}\left|\psi_{\mathbf{A}}\right|\left(\sum_{j=p+1}^{m} \frac{1}{A^{j}}\right)\right) .
$$

Объединяя эти оценки, получаем, что при достаточно больших $A^{j}$

$$
\left|S_{\alpha, \beta, \varnothing}\right| \leqslant C(m, f, \delta, B)\left(\frac{1}{\prod_{j=p+1}^{m} \Lambda\left(\left\lfloor\varkappa^{j} A^{j}\right\rfloor-1\right)}+\sum_{j=p+1}^{m} \frac{1}{A^{j}}\right) \leqslant \varepsilon .
$$

4. Рассмотрим оставшееся слагаемое $(\beta=\gamma=\varnothing)$. Пусть $g(\mathbf{t})=f(\mathbf{t})-f(+0)$. Имеем

$$
\begin{aligned}
\int_{(0, \delta)^{m}} f(\mathbf{t}) \prod_{j=1}^{m} \frac{\sin A^{j} t^{j}}{t^{j}} d \mathbf{t}= & \int_{(0, \delta)^{m}} g(\mathbf{t}) \prod_{j=1}^{m} \frac{\sin A^{j} t^{j}}{t^{j}} d \mathbf{t} \\
& +f(+0) \int_{(0, \delta)^{m}} \prod_{j=1}^{m} \frac{\sin A^{j} t^{j}}{t^{j}} d \mathbf{t} .
\end{aligned}
$$

$\mathrm{K}$ первому слагаемому применим лемму $5:$ поскольку $\lim _{t^{j} \rightarrow+0} g(\mathbf{t})=0$, то с учетом леммы 1 получаем оценку

$$
\left|\int_{(0, \delta)^{m}} g(\mathbf{t}) \prod_{j=1}^{m} \frac{\sin A^{j} t^{j}}{t^{j}} d \mathbf{t}\right| \leqslant C(m)\left(V_{H}\left(g ;(0, \delta)^{m}\right)+\sup _{(0, \delta)^{m}}|g|\right) \leqslant C(m) \varepsilon .
$$

Второе слагаемое распадается в произведение одномерных интегралов Фурье от функций $\chi_{(0, \delta)}(t)$, поэтому (например, по теореме Дирихле-Жордана)

$$
f(+0) \int_{(0, \delta)^{m}} \prod_{j=1}^{m} \frac{\sin A^{j} t^{j}}{t^{j}} d t \rightarrow \frac{1}{2^{m}} f(+0)=f^{*}(0)
$$

при увеличении $A^{j}$.

Таким образом, при достаточно больших $A^{j}$ каждое слагаемое в (13) не превосходит $C(m) \varepsilon$, кроме одного слагаемого (рассмотренного в п. 4$)$, которое сколь угодно близко к $f^{*}(0)$. Теорема доказана. 
ДОКАЗАТЕЛЬСТВО ТЕОРЕМЫ 2. Пусть функция $f(\mathbf{x})$ принадлежит классу $\operatorname{HBV}\left((-\pi, \pi]^{m}\right)$. Без ограничения обшности рассмотрим поведение ряда Фурье в нуле.

Введем вспомогательную функцию

$$
g(\mathbf{x})= \begin{cases}f(\mathbf{x}) \prod_{j=1}^{m} \frac{x^{j}}{2 \sin \left(x^{j} / 2\right)}, & x \in(-\pi, \pi]^{m}, \\ 0, & x \in \mathbb{R}^{m} \backslash(-\pi, \pi]^{m} .\end{cases}
$$

Для $\mathbf{A} \in \mathbb{N}^{m}$ положим $\mathbf{A}^{\prime}=\left(A^{1}+1 / 2, \ldots, A^{m}+1 / 2\right)$. Заметим, что по лемме 2 вьполнено условие $g(\mathbf{x}) \in \mathrm{HBV}\left(\mathbb{R}^{m}\right)$. Кроме того, нетрудно видеть, что по той же лемме

$$
\lim _{\|\boldsymbol{\delta}\| \rightarrow+0} \sum_{\boldsymbol{\sigma} \in\{-1,1\}^{m}} V_{H}(f-g ;(0, \boldsymbol{\sigma} \boldsymbol{\delta}))=0,
$$

т. е. из условий теоремы и неравенства (5) следует, что

$$
\lim _{\|\boldsymbol{\delta}\| \rightarrow+0} \sum_{\boldsymbol{\sigma} \in\{-1,1\} m} V_{H}(f ;(0, \boldsymbol{\sigma} \boldsymbol{\delta}))=0 .
$$

Теперь утверждение теоремы 2 непосредственно вытекает из равенств $f^{*}(0)=$ $g^{*}(0), S_{\mathbf{A}}(f, 0)=\sigma_{\mathbf{A}^{\prime}}(g, 0)$ и теоремы 1 (условие (Б) в ней автоматически выполнено для $B=\pi)$.

\section{§5. Доказательство теорем о расходимости}

Нам понадобится следующая конструкция.

Пусть $m \geqslant 3$. Рассмотрим систему вложенных в $\mathbb{R}$ интервалов $\left\{D_{i}^{1}\right\}_{i=1}^{\infty}$, не образуюших в объединении всё $\mathbb{R}$, и системы попарно непересекающихся интервалов $\left\{D_{i}^{q}\right\}_{i=1}^{\infty}, q=2, \ldots, m$, где $D_{i}^{q}=\left(a_{i}^{q}, b_{i}^{q}\right) \subset \mathbb{R}$. Пусть $f_{i}(x)$ - произвольные функции на $\mathbb{R}$, удовлетворяюшие условию $f_{i}(t)=0$ при $t \leqslant a_{i}^{1}$ и $t \geqslant b_{i}^{1}$.

Обозначим через $h_{i}^{q}(t)$ произвольную функцию, равную нулю при $t \leqslant a_{i}^{q}$ и $t \geqslant b_{i}^{q}$, такую, что $h_{i}^{q}\left(\left(a_{i}^{q}+b_{i}^{q}\right) / 2\right)= \pm 1,\left|h_{i}^{q}(t)\right|$ не убьвает на $\left[a_{i}^{q},\left(a_{i}^{q}+b_{i}^{q}\right) / 2\right]$ и не возрастает на $\left[\left(a_{i}^{q}+b_{i}^{q}\right) / 2, b_{i}^{q}\right]$.

Назовем диагональной функцию на $\mathbb{R}^{m}$, имеющую вид

$$
f(\mathbf{x})=\sum_{i=1}^{\infty}\left(f_{i}\left(x^{1}\right) \prod_{q=2}^{m} h_{i}^{q}\left(x^{q}\right)\right) .
$$

Ясно, что носитель этой функции содержится в объединении замыканий попарно непересекаюшихся параллелепипедов $D_{i}^{1} \times \cdots \times D_{i}^{m}$.

ЛЕмма 7. Пусть последовательности $\Lambda^{2}, \ldots, \Lambda^{m} \in \mathbb{L}$ таковы, ито выполнено условие (4). Тогда следующие условия эквивалентны:

(а) диагональная функиия $f(\mathbf{x})$, определенная формулой $(14)$, принадлежит классу $\left(\Lambda^{1}, \ldots, \Lambda^{m}\right) \mathrm{BV}\left(\mathbb{R}^{m}\right)$;

(б) при всех натуральных $і$ выполнено $f_{i} \in \Lambda^{1} \mathrm{BV}(\mathbb{R})$ и величины $V_{\Lambda^{1}}\left(f_{i} ; \mathbb{R}\right)$ ограничены в совокупности некоторым числом $C$.

Эта лемма доказана в работе автора [5] для функций на $[-\pi, \pi)^{m}$. Из доказательства нетрудно видеть, что лемма справедлива и в приведенном здесь виде. 
ДОКАЗАТЕЛЬСТВО ТЕОРЕМЫ 3. Пусть $n_{k}$-натуральные числа, причем $n_{1}=4$, $n_{k} \geqslant 3, N_{0}=1, N_{k}=n_{1} n_{2} \cdots n_{k}=N_{k-1} n_{k}$. Введем для натуральных $k$ интервалы $D_{k}^{1}=\left(\frac{\pi}{N_{k}}, \frac{\pi}{N_{k-1}}\right)$. Определим функции

$$
f_{k}(t)= \begin{cases}\frac{1}{\ln n_{k}} \sin \left(N_{k} t\right), & t \in D_{k}^{1}, \\ 0, & t \leqslant \frac{\pi}{N_{k}} \text { или } t \geqslant \frac{\pi}{N_{k-1}} .\end{cases}
$$

Для $q=2, \ldots, m$ возьмем $D_{k}^{q}=\left(\frac{\pi}{N_{k}}, \frac{2 \pi}{N_{k}}\right)$ и $h_{j}(t)=h_{k}^{q}(t)=\sin \left(N_{k} t\right)$ на интервале $D_{k}^{q}$ и $h_{j}(t)=0$ вне него.

Тогда диагональная функция

$$
f(\mathbf{x})=\sum_{j=1}^{\infty}\left(f_{2 j}\left(x^{1}\right) \prod_{q=2}^{m} h_{2 j}\left(x^{q}\right)\right)
$$

принадлежит классу $\left(H, \Lambda^{2}, \ldots, \Lambda^{m}\right) \mathrm{BV}\left(T^{3}\right)$ по лемме 7 .

Аналогично [5, теорема 5] доказывается, что при надлежащем выборе последовательности $\left\{n_{k}\right\}$ функция $f(\mathbf{x})$ удовлетворяет всем остальным условиям теоремы.

ДОКАЗАТЕЛЬСТВо ТЕОРЕМЫ 4 . Пусть $A_{k}=4^{k}$; фактически понадобится лишь то, чтобы $A_{k}$ были натуральными числами, $A_{k+1} \geqslant 4 A_{k}$ и $A_{1}>1$. Положим

$$
c_{0}=\int_{\pi}^{2 \pi} \frac{\sin ^{2} t}{t} d t
$$

Будем также пользоваться тем, что при $a>0$ по второй теореме о среднем справедливо неравенство

$$
\left|\int_{a}^{b} \frac{\cos t}{t} d t\right| \leqslant \frac{2}{a}
$$

Определим функции

$$
h_{k}(t)=\left\{\begin{array}{ll}
\sin A_{k} t, & \frac{\pi}{A_{k}} \leqslant t \leqslant \frac{2 \pi}{A_{k}}, \\
0 & \text { иначе },
\end{array} \quad g_{k}(t)= \begin{cases}\sin A_{k} t, & \pi \leqslant t \leqslant \pi A_{k}, \\
0 & \text { иначе. }\end{cases}\right.
$$

Заметим, что $V_{H}\left(g_{k} ; \mathbb{R}\right)<\sum_{j=1}^{A_{k}\left(A_{k}-1\right)+1} \frac{2}{j} \leqslant C \ln A_{k}$. Поэтому если положить

$$
\varphi_{k}(\mathbf{x})=\frac{g_{k}\left(x^{1}\right)}{\ln A_{k}} \prod_{j=2}^{m} h_{k}\left(x^{j}\right), \quad f(\mathbf{x})=\sum_{k=1}^{\infty} \varphi_{k}(\mathbf{x}),
$$

то функция $f(\mathbf{x})$ будет принадлежать классу $\left(H, \Lambda^{2}, \ldots, \Lambda^{m}\right) \mathrm{BV}\left(\mathbb{R}^{m}\right)$ по лемме 7 .

Через $\sigma_{A}(f, \mathbf{x})$ обозначим частичный интеграл Фурье по кубу $[-A, A]^{m}$.

Установим теперь необходимые свойства функции $f(\mathbf{x})$.

(А) Функция $f$ интегрируема по Лебегу на $\mathbb{R}^{m}$. Действительно, носитель $\varphi_{k}(\mathbf{x})$ имеет меру, не большую, чем $\frac{\pi^{m}}{\left(A_{k}\right)^{m-2}}$, и функция $f$ на нем не превосходит $\frac{1}{\ln A_{k}}$, а ряд $\sum \frac{1}{\left(A_{k}\right)^{m-2} \ln A_{k}}$ сходится. 
(Б) При $k \geqslant 3$ выполнено неравенство

$$
\sigma_{A_{k}}\left(\varphi_{k}, 0\right) \geqslant \frac{\left(c_{0}\right)^{m-1}}{4 \pi^{m}}
$$

Действительно,

$$
\begin{aligned}
\sigma_{A_{k}}\left(\varphi_{k}, 0\right) & =\frac{1}{\pi^{m} \ln A_{k}} \int_{\pi}^{A_{k} \pi} \frac{\sin ^{2} A_{k} t}{t} d t\left(\int_{\pi / A_{k}}^{2 \pi / A_{k}} \frac{\sin ^{2} A_{k} s}{s} d s\right)^{m-1} \\
& =\frac{\left(c_{0}\right)^{m-1}}{\pi^{m} \ln A_{k}}\left(\int_{\pi}^{A_{k} \pi} \frac{d t}{2 t}-\int_{\pi}^{A_{k} \pi} \frac{\cos 2 A_{k} t}{2 t} d t\right) \\
& =\frac{\left(c_{0}\right)^{m-1}}{2 \pi^{m}}-\frac{\left(c_{0}\right)^{m-1}}{2 \pi^{m} \ln A_{k}} \int_{2 A_{k} \pi}^{2\left(A_{k}\right)^{2} \pi} \frac{\cos s}{s} d s \\
& \geqslant \frac{\left(c_{0}\right)^{m-1}}{2 \pi^{m}}-\frac{\left(c_{0}\right)^{m-1}}{\pi^{m+1} A_{k} \ln A_{k}}
\end{aligned}
$$

откуда получаем требуемое утверждение.

(В) Докажем, что если $A \leqslant \frac{1}{2} A_{k+1}$, то

$$
\sum_{j=k+1}^{\infty}\left|\sigma_{A}\left(\varphi_{j}, 0\right)\right| \leqslant \frac{1}{A_{k+1}} .
$$

Заметим, что для любых $A$ и $A_{j}$

$$
\left|\int_{\pi / A_{j}}^{2 \pi / A_{j}} \sin A t \frac{\sin A_{j} t}{t} d t\right| \leqslant \pi .
$$

Поэтому

$$
\begin{gathered}
\sum_{j=k+1}^{\infty}\left|\sigma_{A}\left(\varphi_{j}, 0\right)\right| \leqslant \sum_{j=k+1}^{\infty} \frac{1}{\pi^{m} \ln A_{j}}\left|\int_{\pi}^{A_{j} \pi} \frac{\sin A t \sin A_{j} t}{t} d t\right| \cdot \pi^{m-1} \\
\leqslant \sum_{j=k+1}^{\infty} \frac{1}{\pi \ln A_{j}}\left(\frac{1}{2}\left|\int_{\pi\left(A_{j}+A\right)}^{\pi A_{j}\left(A_{j}+A\right)} \frac{\cos s}{s} d s\right|\right. \\
\left.+\frac{1}{2}\left|\int_{\pi\left(A_{j}-A\right)}^{\pi A_{j}\left(A_{j}-A\right)} \frac{\cos s}{s} d s\right|\right)
\end{gathered}
$$

Учитывая оценку (16) и неравенство $A_{j+1} \geqslant 4 A_{j}$, получим

$$
\begin{aligned}
\sum_{j=k+1}^{\infty}\left|\sigma_{A}\left(\varphi_{j}, 0\right)\right| & \leqslant \sum_{j=k+1}^{\infty} \frac{1}{\pi \ln A_{j}}\left(\frac{1}{\pi\left(A_{j}+A\right)}+\frac{1}{\pi\left(A_{j}-A\right)}\right) \\
& \leqslant \sum_{j=k+1}^{\infty} \frac{3}{\pi^{2} A_{j} \ln A_{j}} \leqslant \frac{1}{A_{k+1}}
\end{aligned}
$$

что и требовалось доказать. 
(Г) Наконец, учитывая (19) и (16), аналогично предыдушему получаем, что при $A \geqslant 2 A_{k-1}$

$$
\sum_{j=1}^{k-1}\left|\sigma_{A}\left(\varphi_{j}, 0\right)\right| \leqslant \sum_{j=1}^{k-1} \frac{1}{\pi \ln A_{j}}\left|\int_{\pi}^{A_{j} \pi} \frac{\sin A t \sin A_{j} t}{t} d t\right| \leqslant \frac{3}{\pi A} \sum_{j=1}^{k-1} \frac{1}{\ln A_{j}} \leqslant \frac{C \ln k}{A}
$$

Теперь, с одной стороны, объединяя оценки (17), (18) и (20), получаем, что при достаточно больших $k$ выполнены неравенства

$$
\left|\sigma_{A_{k}}(f, 0)\right| \geqslant \frac{\left(c_{0}\right)^{m-1}}{4 \pi^{m}}-\frac{1}{4^{k+1}}-\frac{C \ln k}{4^{k}}>C(m)>0 .
$$

С другой стороны, для $B_{k}=2 \cdot 4^{k}$ из оценок (18) и (20) получаем, что при $k \rightarrow \infty$

$$
\left|\sigma_{B_{k}}(f, 0)\right| \leqslant \frac{1}{4^{k+1}}+\frac{C \ln k}{2 \cdot 4^{k}} \rightarrow 0 .
$$

Теорема доказана.

Автор благодарен профессору М.И. Дьяченко за полезные замечания.

\section{Список литературы}

1. Waterman D. On convergence of Fourier series of functions of generalized bounded variation // Studia Math. 1972. V. 44. № 1. P. 107-117.

2. Саакян А. А. О сходимости двойных рядов Фурье функций ограниченной гармонической вариации // Изв. АН АрмССР. 1986. Т. 21. №6. С. 517-529.

3. Саблин А. И. Функции ограниченной $\Lambda$-вариации и ряды Фурье: Дис. $\ldots$ канд. физ.матем. наук. М.: МГУ, 1987.

4. Саблин А. И. А-вариация и ряды Фурье // Изв. вузов. Математика. 1987. № 10. С. 66-68.

5. Бахвалов А.H. Непрерывность по $\Lambda$-вариации функций многих переменных и сходимость кратных рядов Фурье // Матем. сб. 2002. Т. 193. № 12. С. 3-20.

6. Зигмунд А. Тригонометрические ряды. Т. 2. М.: Мир, 1965.

7. Бахвалов А.Н. О представлении непериодических функций ограниченной $\Lambda$-вариации интегралом Фурье // Вестн. МГУ. Сер. 1. Математика. Механика. 1998. № 3. С. 6-12.

8. Драгошанский O.C. О непрерывности двумерной $\Lambda$-вариации // Современные проблемы теории функций и их приложения: Тез. докл. 11-й Саратовской зимней школы. Саратов: Изд-во ГосУНЦ "Колледж", 2002. С. 71-72.

9. Goffman C., Waterman D. The localization principle for Fourier series // Studia Math. 1980. V. 99. № 1. P. 41-57.

10. Schramm M., Waterman D. On the magnitude of Fourier coefficients // Proc. Amer. Math. Soc. 1982. V. 85. № 3. P. 408-410.

Московский государственный университет

Поступило в редакцию им. М. В. Ломоносова

17.XII. 2002

E-mail: alex@abs.math.msu.su 